Supporting Information

\title{
Ordered Surface Nanostructures Self-Assembled from Rod-Coil Block Copolymers on Microspheres
}

Wenheng $\mathrm{Xu}$, Zhanwen $\mathrm{Xu}$, Chunhua Cai*, Jiaping Lin*, Shengmiao Zhang, Liangshun Zhang, Shaoliang Lin, Yuan Yao and Huimin Qi

Shanghai Key Laboratory of Advanced Polymeric Materials, Key Laboratory for Ultrafine Materials of Ministry of Education, School of Materials Science and Engineering, East China University of Science and Technology, Shanghai 200237, China

*Corresponding authors. E-mail address: caichunhua@ecust.edu.cn, jlin@ecust.edu.cn 


\section{Contents}

1. Synthesis of Polymers and Microspheres ...............................................................

1.1 Materials and regents ......................................................................................................

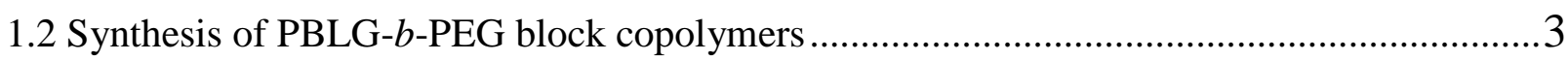

1.3 Synthesis of PS-b-PEG block copolymers.........................................................................

1.4 Synthesis of PS microspheres ...................................................................................

2. Preparation of Surface-Patterned Microparticles.....................................................6

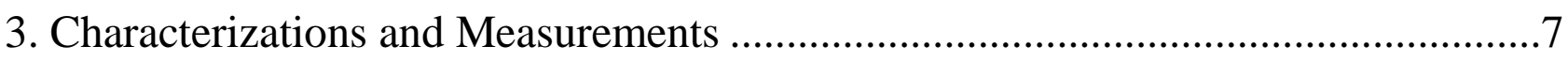

4. Characterizations of Block Copolymers and PS Microspheres.................................8

5. Effect of Rigidity of the Building Blocks on Surface Nanostructure......................11

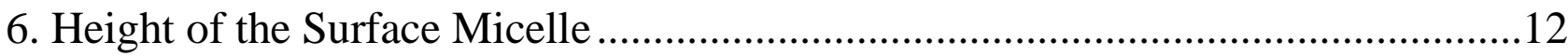

7. Effect of Molecular Weight of PBLG Blocks on Surface Nanostructure.................14

8. Statistical Method for Analysing the Array Feature of Surface Micelles.................16

9. Array Feature of Surface Micelles ..................................................................17

10. Simulation Method and Parameter Setting ........................................................20

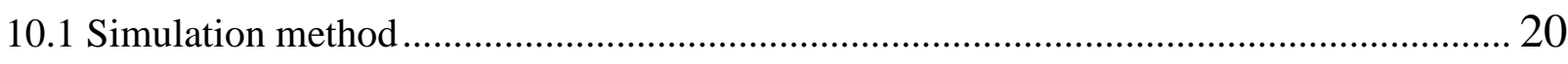

10.2 Simulation parameters ……………………………………………………….... 21

11. Simulations for Effect of Rod Block Length on Surface Nanostructure ................23 


\section{Synthesis of Polymers and Microspheres}

\subsection{Materials and regents}

$\alpha$-Methoxy- $\omega$-amino poly(ethylene glycol) (mPEG-NH ${ }_{2}, M_{\mathrm{n}}=5 \mathrm{~kg} \cdot \mathrm{mol}^{-1}$ ) was purchased from Sigma-Aldrich. $\quad \gamma$-Benzyl-L-glutamate-N-carboxyanhydride (BLG-NCA) was synthesized according to literatures. ${ }^{1-3}$ mPEG-Br was synthesized by the esterification of mPEG-OH and 2-bromo-2-methylpropionyl bromide. ${ }^{4,5}$ Styrene (St, Aldrich) was distilled before use. Divinylbenzene (DVB, 80\%, Aldrich) was purified by passing through neutral chromatographic aluminum oxide to eliminate the inhibitor. Ammonium persulfate (APS) was recrystallized twice in deionized water before use. Copper(I) bromide (CuBr, Sigma-Aldrich, 98\%) was purified by stirring overnight with acetic acid at room temperature, filtrated, followed by washing with ethanol, diethyl ether, and acetone prior to drying under vacuum for 1 day. Other chemicals were obtained from Adamas-beta, and purified according to conventional methods or used as received. Deionized water was prepared in a Millipore Super-Q Plus Water System to a level of $18.2 \mathrm{M} \Omega \cdot \mathrm{cm}$ resistance. Dialysis bag (Membra-cel, 3500 molecular weight cutoff) was provided by Serva Electrophoresis GmbH.

\subsection{Synthesis of PBLG-b-PEG block copolymers}

Poly( $\gamma$-benzyl-L-glutamate)-block-poly(ethylene glycol) (PBLG-b-PEG) rod-coil block copolymers (BCPs) were synthesized by ring-opening polymerization of BLG-NCA in anhydrous 1,4-dioxane initiated by mPEG-NH 2 (macroinitiator) according to our previous work. $^{1-3}$ To remove trace amounts of water in initiator, mPEG-NH 2 was dissolved in toluene and then removing the toluene/water in high vacuum before use. The reaction was performed 
in flame-dried reaction bottle under a dry nitrogen atmosphere for 3 days at $15{ }^{\circ} \mathrm{C}$. The resulting products were purified twice by repeated precipitation from a chloroform solution into a large volume of anhydrous methanol.

\subsection{Synthesis of PS-b-PEG block copolymers}

Polystyrene-block-poly(ethylene glycol) (PS-b-PEG) coil-coil BCPs were synthesized by atom transition free radical polymerization (ATRP). ${ }^{4,5}$ Firstly, the mPEG-Br and styrene were added to a $50 \mathrm{~mL}$ Schlenk flask followed by addition of solvent (anisole). Next, PMDETA and $\mathrm{CuBr}$ were introduced and stirred together for $30 \mathrm{~min}$. The solution was degassed by three cycles of freeze-pump-thaw to remove any dissolved oxygen and purged with dry nitrogen. The mixture was stirred at $90{ }^{\circ} \mathrm{C}$. After $5 \mathrm{~h}$, the reaction mixture was dissolved in THF and then passed through an $\mathrm{Al}_{2} \mathrm{O}_{3}$ column to remove the copper catalyst. The resulting solution was concentrated by rotary evaporation, and then was poured into a large volume of anhydrous methanol. The precipitated product was dried under vacuum and then purified twice by repeated precipitation from a THF solution into a large volume of anhydrous methanol. Finally, after vacuum drying, PS-b-PEG BCPs were successfully obtained. The detailed information of PS- $b$-PEG coil-coil BCPs is provided in Table S1.

\subsection{Synthesis of PS microspheres}

The polystyrene (PS) microspheres used in this work were prepared with surfactant-free emulsion copolymerization of St. $^{6,7}$ Water containing APS and DVB were added to a three-necked glass reactor with an overhead leaf-shaped stirrer paddle and a condenser. Then the St monomer was added to the reactor. The reactor was purged nitrogen before elevating 
the temperature, and the nitrogen blanket was maintained throughout the polymerization. The mixtures were stirred steadily at $230 \mathrm{rpm}$ and left at $70^{\circ} \mathrm{C}$ for $12 \mathrm{~h}$, before it was cooled to room temperature. The polymer emulsion was separated by centrifugation to remove the liquid supernatant, adding deionized water into the system and dispersed by ultrasonic machine. The dispersed sample was settled by centrifugal machine. Repeated washing with deionized water to ensure removal of unreacted reactants. Finally, after vacuum drying, PS microspheres were obtained. 


\section{Preparation of Surface-Patterned Microparticles}

Surface-patterned microspheres were fabricated by the adsorption-assembly of PBLG- $b$-PEG rod-coil BCPs on the surface of PS microspheres. First, PBLG- $b$-PEG rod-coil BCPs and PS microspheres were dissolved/dispersed in THF/DMF mixtures. In the stock solution, the concentrations of the BCPs and PS microspheres were same $0.5 \mathrm{~g} \cdot \mathrm{L}^{-1}$. Then, 1 mL PBLG- $b$-PEG BCP solutions and 3 mL PS microsphere dispersions were mixed together. To the solution, $1.6 \mathrm{~mL}$ deionized water, a selective solvent for PEG blocks, was dropped at a rate of $c a .0 .2 \mathrm{~mL} \cdot \mathrm{min}^{-1}$ with stirring. With the addition of water, the PBLG-b-PEG rod-coil BCPs were first adsorbed on the surface of PS microspheres. The BCPs then self-assembled into ordered surface nanostructures on PS microspheres. After being stabilized for about 30 min, the solution was dialyzed against deionized water for 3 days to remove organic solvents. After dialysis, aqueous solution of PS microspheres with surface micelles was obtained. 


\section{Characterizations and Measurements}

Nuclear Magnetic Resonance (NMR). The molecular weight of BCPs was estimated using $400 \mathrm{MHz}{ }^{1} \mathrm{H}$ NMR measurement (Avance 550, Bruker) in the solvent of $\mathrm{CDCl}_{3}$ and trifluoroacetic acid (TFA) mixture at $25{ }^{\circ} \mathrm{C}$.

Gel Permeation Chromatography (GPC). The molecular weight distributions (PDI) of BCPs were obtained from GPC testing (PL 50 plus, DMF as eluent solvent).

Scanning Electron Microscopy (SEM). The surface profile of the aggregates was obtained from Field Emission SEM (S4800, Hitachi) operated at an accelerating voltage of 15 $\mathrm{kV}$. The sample was prepared by placing drops of solution on a Si Wafer and then was dried at room temperature. Before observation, the samples were sputtered by gold.

Atom Force Microscopy (AFM). AFM images were obtained with XE-100 AFM instrument (Park Systems), employing the tapping mode. The samples were prepared by placing one drop of solution on a fresh-cleaved mica surface, and the sample was allowed to be dried in air. 


\section{Characterizations of Block Copolymers and PS Microspheres}

For PBLG- $b$-PEG BCPs, since the molecular weight of the PEG block is known $\left(M_{\mathrm{n}}=5\right.$ $\left.\mathrm{kg} \cdot \mathrm{mol}^{-1}\right)$, the number-average molecular weight $\left(M_{\mathrm{n}}\right)$ and the degree of polymerization (DP) of PBLG blocks can be calculated from the intensity ratio of the methylene proton signal (5.1 ppm) of polypeptide to the ethylene proton signal (3.6 ppm) of PEG. For PS- $b$-PEG BCPs, the molecular weight of PS block is calculated from the intensity ratio of the phenyl ortho proton signal (6.3-6.9 ppm) of PS to the ethylene proton signal (3.6 ppm) of PEG. The PDI values of these polymers were determined by GPC analysis. The detailed information of PBLG- $b$-PEG and PS- $b$-PEG BCPs is provided in Figure S1 and Table S1.
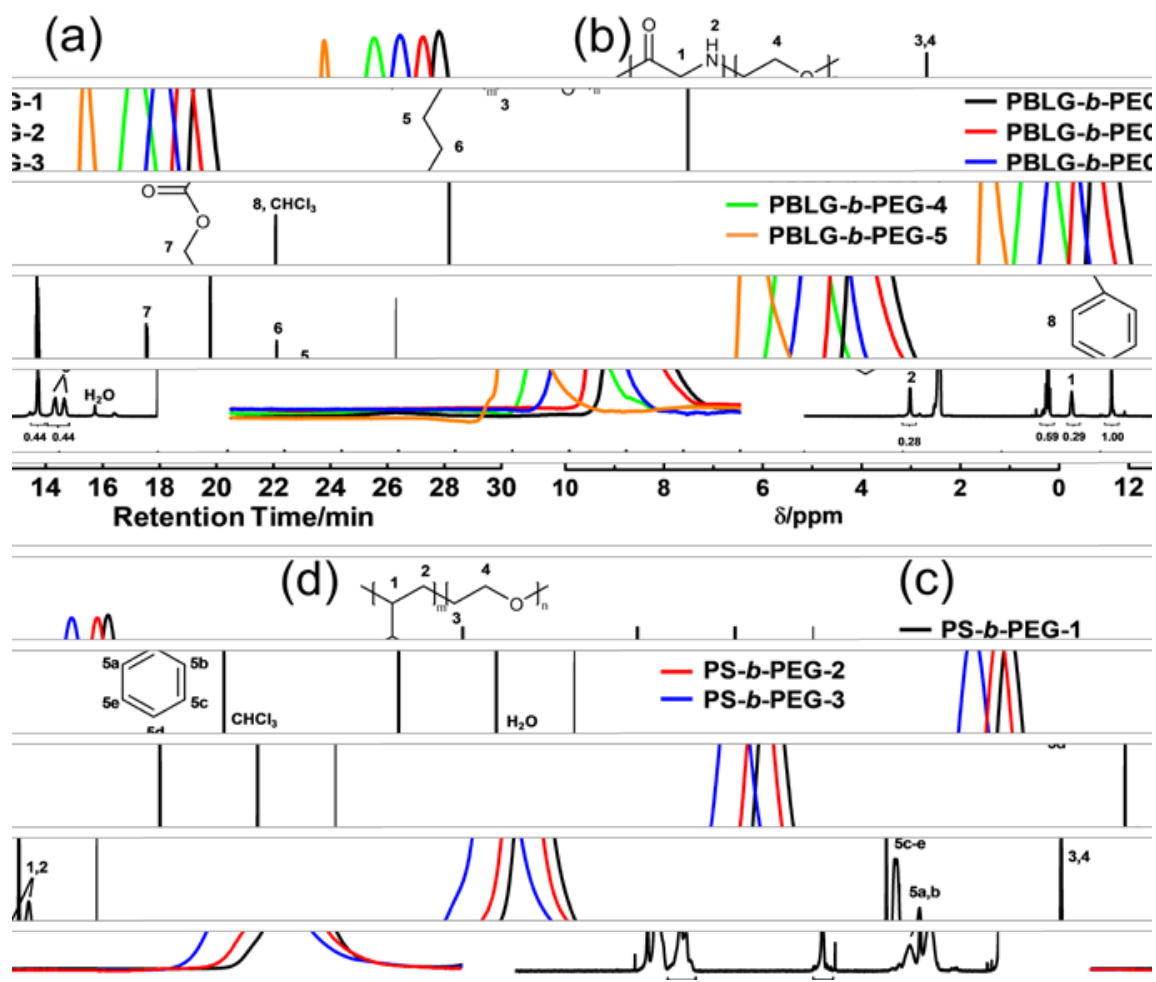

Figure S1. (a) GPC curves of PBLG-b-PEG BCPs, and (b) NMR spectrum of PBLG $_{20.1 \mathrm{k}}-b-\mathrm{PEG}_{5 \mathrm{k}}$; (c) GPC curves of PS- $b$-PEG BCPs, and (d) NMR spectrum of $\mathrm{PS}_{15.5 \mathrm{k}}-b-\mathrm{PEG}_{5 \mathrm{k}}$. 
Table S1. Characterization of block copolymers

\begin{tabular}{cccc}
\hline Polymer & $\begin{array}{c}M_{\mathrm{n}}{ }^{\mathrm{a}} \text { of block copolymers } \\
\left(\mathrm{kg} \cdot \mathrm{mol}^{-1}\right)\end{array}$ & $\begin{array}{c}\text { DP }{ }^{\mathrm{a}} \text { of PBLG } \\
\text { or PS blocks }\end{array}$ & PDI $^{\mathrm{b}}$ \\
\hline PBLG $_{15.3 \mathrm{k}}-b-\mathrm{PEG}_{5 \mathrm{k}}$ & 20.3 & PBLG: 70 & 1.17 \\
PBLG $_{20.1 \mathrm{k}}-b-\mathrm{PEG}_{5 \mathrm{k}}$ & 25.1 & PBLG: 92 & 1.20 \\
PBLG $_{29.5 \mathrm{k}}-b-\mathrm{PEG}_{5 \mathrm{k}}$ & 34.5 & PBLG: 135 & 1.19 \\
PBLG $_{39.3 \mathrm{k}}-b-\mathrm{PEG}_{5 \mathrm{k}}$ & 44.3 & PBLG: 179 & 1.16 \\
PBLG $_{61.5 \mathrm{k}}-b-\mathrm{PEG}_{5 \mathrm{k}}$ & 66.5 & PBLG: 281 & 1.17 \\
PS $_{10.3 \mathrm{k}}-b-\mathrm{PEG}_{5 \mathrm{k}}$ & 15.3 & PS: 99 & 1.13 \\
PS $_{15.5 \mathrm{k}-b-\mathrm{PEG}_{5 \mathrm{k}}}$ & 20.5 & PS: 149 & 1.14 \\
PS $_{30.2 \mathrm{k}}-b-\mathrm{PEG}_{5 \mathrm{k}}$ & 35.2 & PS: 290 & 1.13 \\
\hline
\end{tabular}

${ }^{a}$ For PBLG- $b$-PEG and PS- $b$-PEG BCPs, since the $M_{\mathrm{n}}$ value and DP of PEG segment is known, the $M_{\mathrm{n}}$ and DP of PBLG and PS blocks were derived according to ${ }^{1} \mathrm{H}$ NMR spectra in $\mathrm{CDCl}_{3}$.

${ }^{\mathrm{b}}$ The PDI values of the polymers are obtained from GPC testing. 
For the PS microspheres, as shown in Figure S2, they have uniform shape and diameter. The diameter of the PS microspheres is about $609.6 \pm 10.2 \mathrm{~nm}$. The surface of these spheres is smooth, and some uneven parts on the surface were also observed (indicated by red circle in Figure S2).

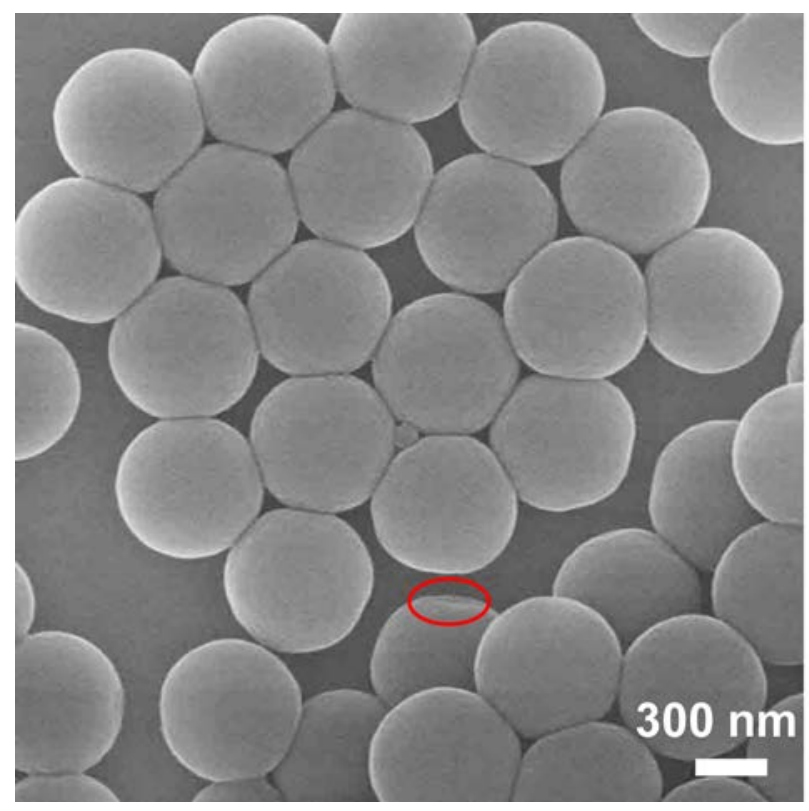

Figure S2. SEM image of PS microspheres synthesized by surfactant-free emulsion copolymerization of St. The red circle show the uneven part on the spherical surface. 


\section{Effect of Rigidity of the Building Blocks on Surface Nanostructure}

The rod-coil nature of the PBLG- $b$-PEG BCPs was found to be essential for preparing these surface micelles. Since the attractions between PBLG/PS is comparable to that between PS/PS, ${ }^{8,9}$ we used PS- $b$-PEG coil-coil BCPs to replace PBLG- $b$-PEG rod-coil BCPs to study the importance of the chain rigidity on the formation of surface micelles. Three PS- $b$-PEG samples with same PEG blocks and various PS block lengths, i.e., $\mathrm{PS}_{10.3 \mathrm{k}}-b-\mathrm{PEG}_{5 \mathrm{k}}$, $\mathrm{PS}_{15.5 \mathrm{k}}-b-\mathrm{PEG}_{5 \mathrm{k}}$ and $\mathrm{PS}_{30.2 \mathrm{k}}-b-\mathrm{PEG}_{5 \mathrm{k}}$ were used in the studies. It was found that PS microspheres can be stabilized by the PS-b-PEG BCPs in water, which indicates the adsorption of PS- $b$-PEG BCPs on PS spheres. However, as shown in Figure S3, homogeneous surface of the PS spheres were obtained, and some small spherical micelles self-assembled from PS-b-PEG BCPs were also observed.

These results indicate that as compared with the coil-coil BCPs, the rod-coil BCPs indeed tend to self-assemble on the surface of microspheres forming ordered nanostructures. These results suggested that the rod-coil nature of the PBLG- $b$-PEG BCPs is essential for preparing the surface micelles.
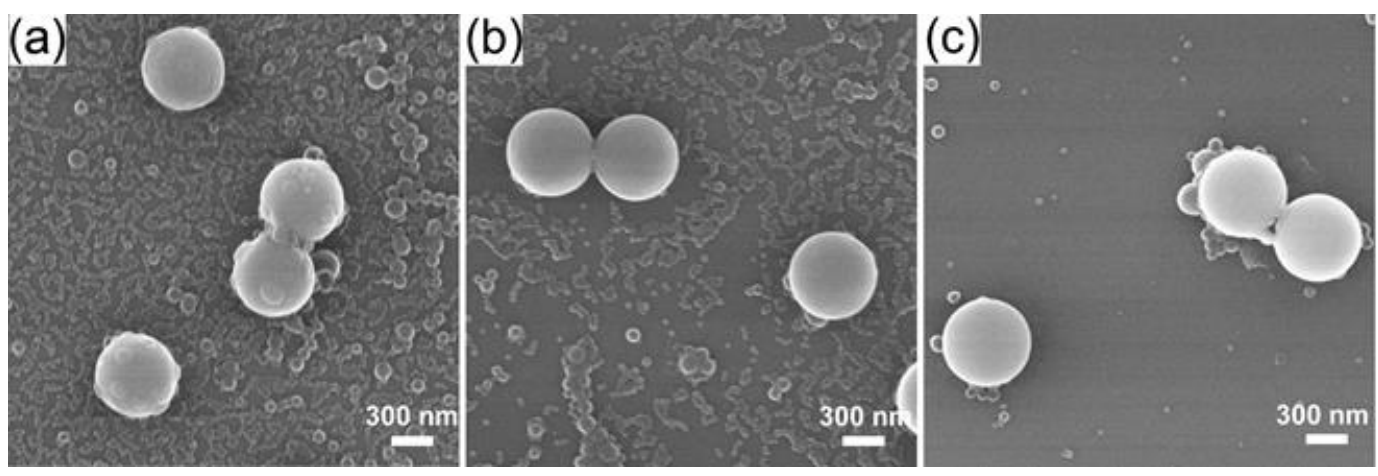

Figure S3. SEM image of aggregates self-assembled from PS microspheres and various PS- $b$-PEG BCPs: (a) PS $10.3 \mathrm{k}-b-\mathrm{PEG}_{5 \mathrm{k}}$; (b) $\mathrm{PS}_{15.5 \mathrm{k}}-b-\mathrm{PEG}_{5 \mathrm{k}}$; (c) $\mathrm{PS}_{30.2 \mathrm{k}}-b-\mathrm{PEG}_{5 \mathrm{k}}$. 


\section{Height of the Surface Micelle}

The height of the surface micelles were obtained from the differences between diameter of the original PS spheres and that of the surface-patterned microparticles. The average diameter and size distribution of the particles are shown in Figure S4. The average diameter of the original PS spheres is $609.6 \pm 10.2 \mathrm{~nm}$ (Figure S4a). For the microparticles with surface micelles formed by $\mathrm{PBLG}_{20.1 \mathrm{k}}-b-\mathrm{PEG}_{5 \mathrm{k}}$, $\mathrm{PBLG}_{29.5 \mathrm{k}}-b-\mathrm{PEG}_{5 \mathrm{k}}$, and $\mathrm{PBLG}_{39.3 \mathrm{k}}-b-\mathrm{PEG}_{5 \mathrm{k}}$ BCPs, as shown in Figure S4b-d, their diameters are $636.2 \pm 11.5 \mathrm{~nm}, 654.5 \pm 4.8 \mathrm{~nm}$ and $673.4 \pm 9.8$ $\mathrm{nm}$, respectively. Therefore, the heights of the surface micelles are about $13.3 \pm 1.7 \mathrm{~nm}$, 22.5 $\pm 1.5 \mathrm{~nm}$, and $31.9 \pm 3.9 \mathrm{~nm}$, respectively. Due to the rigid nature of the PBLG blocks, their length can be estimated from their molecular weights. The length for PBLG $_{20.1 \mathrm{k}}$, $\mathrm{PBLG}_{29.5 \mathrm{k}}$, and $\mathrm{PBLG}_{39.3 \mathrm{k}}$ are approximately $13.8 \mathrm{~nm}, 20.2 \mathrm{~nm}$, and $26.9 \mathrm{~nm}$, respectively. The length of PBLG blocks is approximate with the height of surface micelles (Figure S4e).

The height of the surface micelles should be equal to their radius (half of the diameter), however, in the present system, the height of the surface micelles is slight smaller than radius. The reason could be as follows. Under the SEM testing conditions, the PEG block on the corona of the surface micelles collapse, the obtained height of the surface micelles mainly reflect the core size of the surface micelles attributed by the PBLG blocks. For the diameter of the surface micelles, it is obtained by measuring the distance of the neighboring surface micelles, which could be less affected by the drying process. As a result, the height of the surface micelles is smaller than their radius as obtained from SEM testing. 

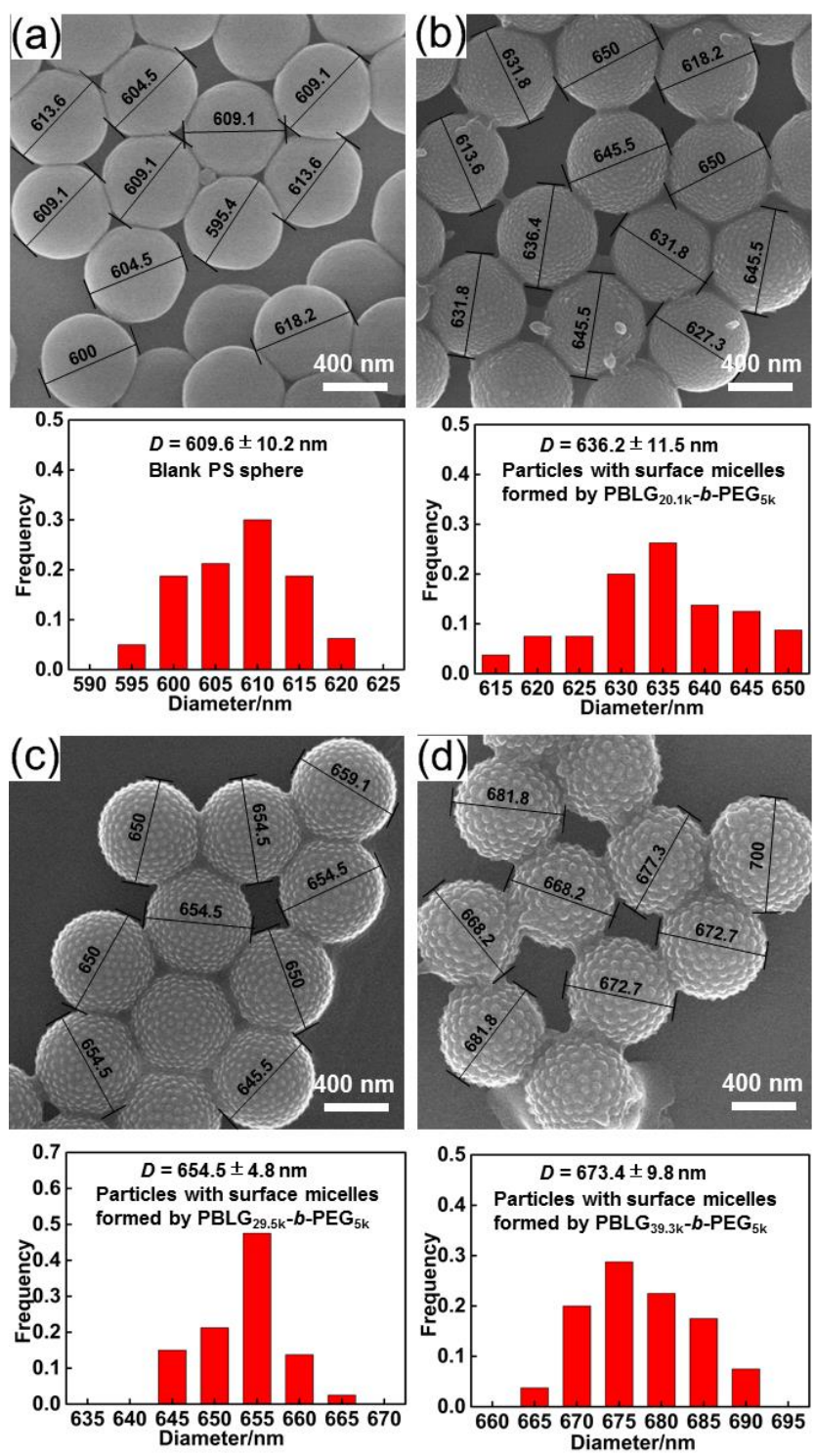

(e)

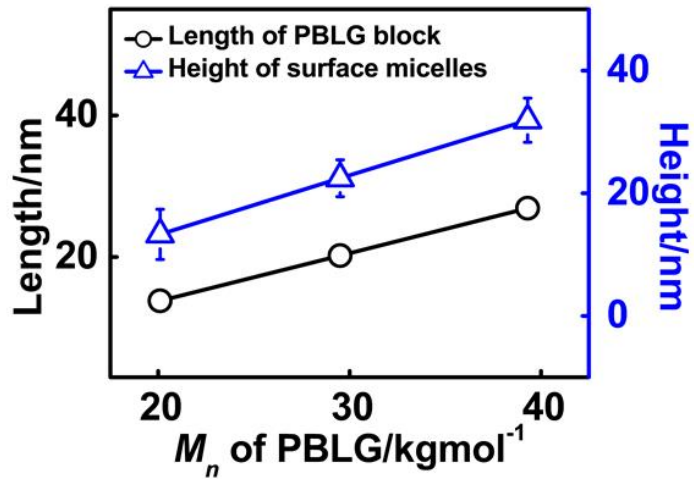

Figure S4. (a) SEM image and size distribution of PS original particles before assembly. (b-d) SEM images and size distributions of surface-patterned microparticles self-assembled from PS spheres and PBLG-b-PEG with various $M_{\mathrm{n}}$ values of the PBLG blocks: (b) $M_{\mathrm{n}}=20.1$ $\mathrm{kg} \cdot \mathrm{mol}^{-1}$; (c) $M_{\mathrm{n}}=29.5 \mathrm{~kg} \cdot \mathrm{mol}^{-1}$; (d) $M_{\mathrm{n}}=39.3 \mathrm{~kg} \cdot \mathrm{mol}^{-1}$. (e) Plots of $M_{\mathrm{n}}$ of the PBLG block vs. height of the surface micelles. 


\section{Effect of Molecular Weight of PBLG Blocks on Surface Nanostructure}

Figure S5 shows the surface-patterned microparticles self-assembled from PS spheres and PBLG- $b$-PEG with various molecular weights of PBLG blocks. As shown in Figure S5a-c, when $M_{\mathrm{n}}$ of PBLG is $20.1 \mathrm{~kg} \cdot \mathrm{mol}^{-1}, 29.5 \mathrm{~kg} \cdot \mathrm{mol}^{-1}$ and $39.3 \mathrm{~kg} \cdot \mathrm{mol}^{-1}$, ordered dot-like surface micelles are formed on microparticles. And the size of the surface micelles increases with the molecular weight of the PBLG blocks. However, when $M_{\mathrm{n}}$ of PBLG is much smaller $\left(M_{\mathrm{n}}=15.3 \mathrm{~kg} \cdot \mathrm{mol}^{-1}\right)$, microspheres with homogeneous surface are obtained (Figure S5d). When $M_{\mathrm{n}}$ of PBLG is much larger $\left(M_{\mathrm{n}}=61.5 \mathrm{~kg} \cdot \mathrm{mol}^{-1}\right)$, microspheres with rough surface were obtained and free micelles formed by the BCPs were also observed (Figure S5e).

The reason for the system does not produce ordered surface nanostructures when the $M_{\mathrm{n}}$ of PBLG blocks is much smaller or much larger than a threshold value can be explained as follows. As stated in the main text, the PBLG-b-PEG block copolymer surface micelles on PS spheres are formed through an adsorption-assembly process with the addition of water. There are two successive processes including adsorption of the PBLG-b-PEG BCPs on PS spheres and subsequent assembly of the BCPs into surface micelles. For the BCPs with a lower molecular weight of PBLG, attraction between PBLG blocks is weak. With the addition of water, the BCPs can adsorb on PS spheres forming a layer, but fail to pack orderly with each other. As a result, no ordered surface micelles are formed. For the BCPs with a larger molecular weight of PBLG, the attraction between PBLG blocks is strong. With the addition of water, most of the BCPs formed stable free micelles in solution, and fewer BCPs adsorb onto the surface of the PS spheres. As a result, no regular surface micelles are observed. 

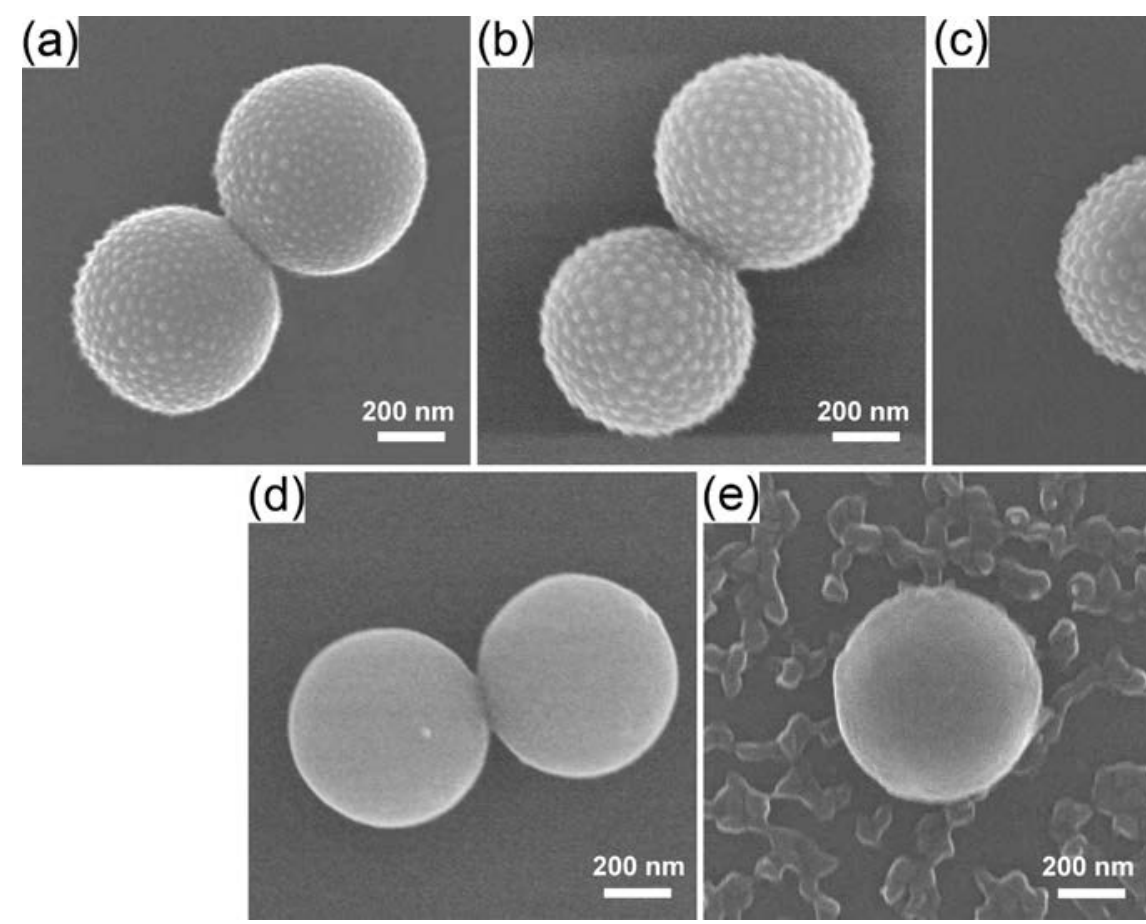

Figure S5. Effect of $M_{\mathrm{n}}$ of PBLG blocks of PBLG- $b$-PEG BCPs on the surface morphologies:

(a) $M_{\mathrm{n}}=20.1 \mathrm{~kg} \cdot \mathrm{mol}^{-1}$; (b) $M_{\mathrm{n}}=29.5 \mathrm{~kg} \cdot \mathrm{mol}^{-1}$; (c) $M_{\mathrm{n}}=39.3 \mathrm{~kg} \cdot \mathrm{mol}^{-1}$; (d) $M_{\mathrm{n}}=15.3$ $\mathrm{kg} \cdot \mathrm{mol}^{-1}$; (e) $M_{\mathrm{n}}=61.5 \mathrm{~kg} \cdot \mathrm{mol}^{-1}$ 


\section{Statistical Method for Analysing the Array Feature of Surface Micelles}

To further understand the array feature of surface micelles, we counted the number of the surface micelles and calculated the defects (5-fold and 7-fold coordinated surface micelles). In general, Delaunay triangulation method has been used to divide the spherical surface into small triangles by connecting every dot-like surface micelle. ${ }^{10,11}$ It is a simple and universal method to examine the number of spherical nanoparticles on microspheres surface. Theoretically, the surface area of one microsphere contains two parts, one is the area of dot-like surface micelles occupied, and another one is the empty space among each three dot-like surface micelles that cannot be occupied by micelle (the inset schematic diagram in Figure S6a).
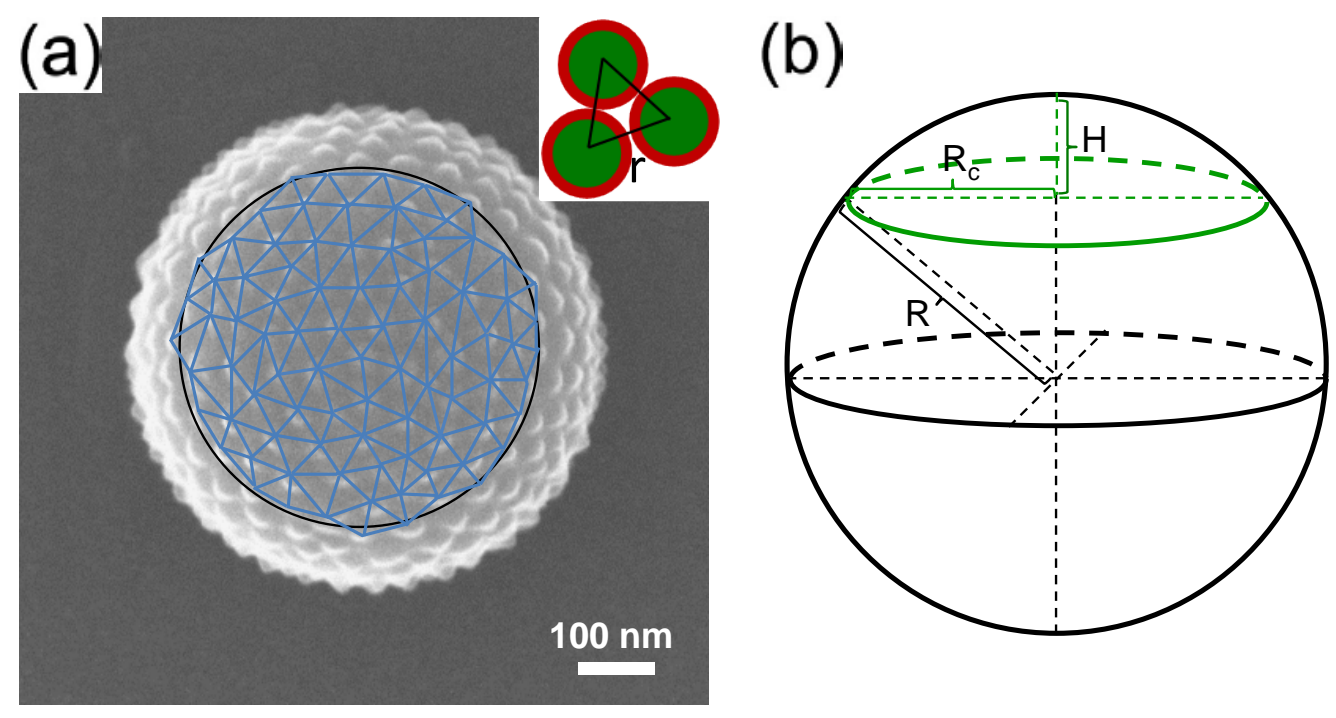

Figure S6. (a) Triangulation graph of spherical surface with dot-like micelles self-assembled by PBLG-b-PEG (the inset is the schematic diagram of empty space among each three dot-like surface micelles); (b) the schematic diagram of the limited area on the spherical surface. 
The area of each dot-like surface micelle and each empty space among three tangential surface micelles on microsphere surface can be calculated by the average radius of dot-like surface micelles (radius $=r$ ). In addition, the number of dot-like surface micelles and empty space of each three surface micelles is related. Since for 2-dimensional topological polyhedron, the Euler characteristic $(\chi)$ can be denoted by the following formula:

$$
\chi=F-E+V
$$

In which $\mathrm{F}, \mathrm{E}, \mathrm{V}$ represents the number of points, edges and faces, respectively. For the spherical structure, the $\chi=2$ and the $E=3 / 2 V$. The formula of the number of points and faces on spherical surface is deduced as following:

$$
V=2(F-2)
$$

For a microsphere of which radius is $\mathrm{R}$, the surface area of it is the area of each dot-like surface micelle plus each empty space among three tangential surface micelles. The formula is defined as:

$$
4 \pi R^{2}=\pi r^{2} F+2(F-2)(2 r)^{2}\left(\frac{\sqrt{3}}{4}-\frac{\pi}{8}\right)
$$

Therefore, the theoretical number of dot-like surface micelles is calculated.

As shown in Figure S6a, since SEM images reflect only top side of the spheres, an extrapolation method was used to deduce the total number of surface micelles on a sphere. We select an effective visible region on the spheres (indicated by a black circle), in which the number and arrangement of the surface micelles can be readily analyzed. In order to deduce the total number, the ratio of the surface area in the black circle to the total surface area should be determined. 
To illustrate how to obtain the ratio of the surface area in the black circle to the total surface area (Figure S6a), we plot a model sphere (Figure S6b). In the model sphere, the surface area of the top sphere crown in the green circle corresponds to the area in the black circle in Figure S6a. The surface area for a sphere crown is $S_{c}=2 \pi R H$ ( $R$ and $H$ represent the radius of the sphere and the height of the spherical crown, respectively). For a sphere, the surface area is $S=4 \pi R^{2}$. Therefore, the ratio for the area of the crown to that of the sphere is $S_{C} / S=H / 2 R$. The $H$ value of the crown the cannot be directly obtained, while the radius of the crown $\left(R_{c}\right)$ can be directly measured. From $R_{c}$ and $R$, the $H$ value can be calculated:

$$
H=R-\left(R^{2}-R_{C}^{2}\right)^{1 / 2}
$$

Therefore, the ratio of the accounting area on a sphere is calculated as:

$$
S_{C} / S=\left(R-\left(R^{2}-R_{C}^{2}\right)^{1 / 2}\right) / 2 R
$$

For the surface patterned sphere shown in Figure S6a, the radius of the sphere $(R)$ is measured to be $304.5 \mathrm{~nm}$, and the radius of the selected crown $\left(R_{\mathrm{c}}\right)$ is $239.9 \mathrm{~nm}$. Therefore, the ratio of the limited area on sphere is about $19.2 \%$. Since the number of triangles in the limited area can be observed, the number of dot-like surface micelles in the limited area is calculated by the equation S3. Finally, by extrapolating to the whole spherical surface, the total number and arrangement of the surface micelles is obtained. 


\section{Array Feature of Surface Micelles}

As shown in Figure S7, surface micelles self-assembled from PBLG $_{20.1 \mathrm{k}}-b-\mathrm{PEG}_{5 \mathrm{k}}$ (Figure S7a,b), PBLG $29.5 \mathrm{k}-b-\mathrm{PEG}_{5 \mathrm{k}}$ (Figure S7c,d) and $\mathrm{PBLG}_{39.3 \mathrm{k}}-b-\mathrm{PEG}_{5 \mathrm{k}}$ (Figure S7e,f) are formed. By accounting the number of surface micelles in the limited area and extrapolating to the whole sphere surface, the average number of surface micelles can be obtained. Array feature analysis revealed that the 6-fold coordination is the majority array type for the surface micelles in all the systems. The total excess disclinations which is the differences between 5-fold coordination (+1 disclination) and 7-fold coordination (-1 disclination) are close to the theoretical value of 12 .
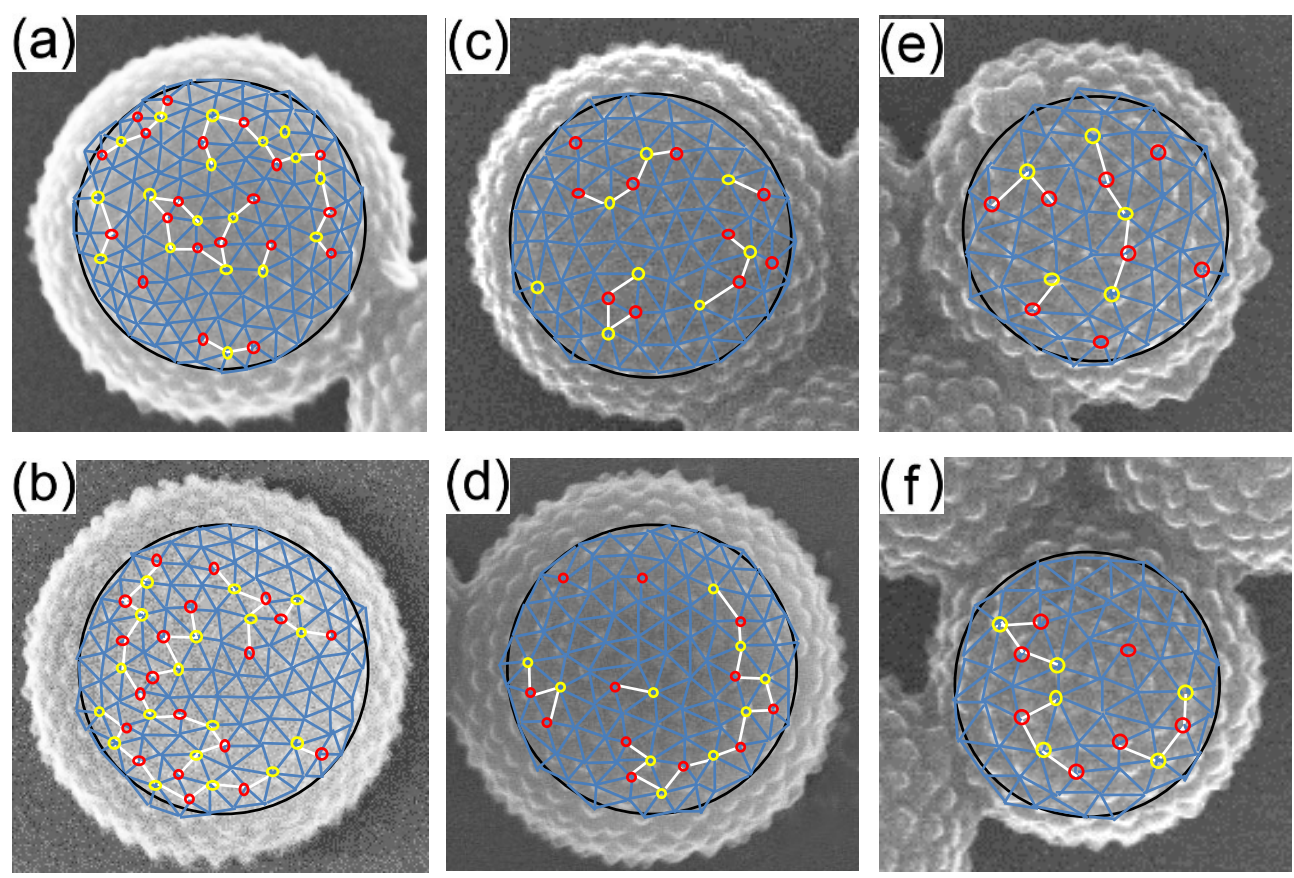

Figure S7. Array feature analysis of the surface micelles form by various PBLG- $b$-PEG BCPs. $M_{\mathrm{n}}$ of the PBLG blocks: (a,b) $20.1 \mathrm{~kg} \cdot \mathrm{mol}^{-1}$; (c,d) $29.5 \mathrm{~kg} \cdot \mathrm{mol}^{-1}$; $(\mathrm{e}, \mathrm{f}) 39.3 \mathrm{~kg} \cdot \mathrm{mol}^{-1}$. The small red and yellow circles represent 5-fold coordinated (+1 disclination) and 7-fold coordinated ( -1 disclination) surface micelles, respectively. 


\section{Simulation Method and Parameter Setting}

\subsection{Simulation method}

The dissipative particle dynamics (DPD) method proposed by Hoogerbrugge and Koelman in 1992, was performed to investigate the self-assembled morphologies. In DPD method, a bead represents the center of mass of a group of monomers. ${ }^{12-14}$ The force acting on each bead includes non-bonded and bonded forces. The non-bonded force acting on a particle, $\mathbf{f}_{\alpha}$, is a pairwise additive force, consisting of the conservative force $\left(\mathbf{F}_{\alpha \beta}^{\mathrm{C}}\right)$, dissipative force $\left(\mathbf{F}_{\alpha \beta}^{\mathrm{D}}\right)$, and random force $\left(\mathbf{F}_{\alpha \beta}^{\mathrm{R}}\right)$. The conservative force is a soft repulsion taking the form as

$\mathbf{F}_{\alpha \beta}^{\mathrm{C}}=a_{\alpha \beta} \sqrt{\omega\left(r_{\alpha \beta}\right)} \hat{\mathbf{r}}_{\alpha \beta}$

where $a_{\alpha \beta}$ is the maximum repulsive interaction between particles $\alpha$ and $\beta, \mathbf{r}_{\alpha \beta}=\mathbf{r}_{\alpha}-\mathbf{r}_{\beta}, r_{\alpha \beta}=\left|\mathbf{r}_{\alpha \beta}\right|$, $\hat{\mathbf{r}}_{\alpha \beta}=\mathbf{r}_{\alpha \beta} / r_{\alpha \beta}$ and $\omega\left(r_{\alpha \beta}\right)$ is the weight function, given by

$$
\omega\left(r_{\alpha \beta}\right)= \begin{cases}\left(1-r_{\alpha \beta} / r_{c}\right)^{2} & \left(r_{\alpha \beta}<r_{c}\right) \\ 0 & \left(r_{\alpha \beta} \geq r_{c}\right)\end{cases}
$$

where $r_{\mathrm{c}}\left(r_{\mathrm{c}}=1.0\right)$ is the cut-off radius. The dissipative force is a friction force that acts on the relative velocities of particles, defined as

$\mathbf{F}_{\alpha \beta}^{\mathrm{D}}=-\gamma \omega^{\mathrm{D}}\left(r_{\alpha \beta}\right)\left(\hat{\mathbf{r}}_{\alpha \beta} \cdot \mathbf{v}_{\alpha \beta}\right) \hat{\mathbf{r}}_{\alpha \beta}$

and the random force, compensating the loss of kinetic energy due to the dissipative force, is defined as

$\mathbf{F}_{\alpha \beta}^{\mathrm{R}}=\sigma \omega^{\mathrm{R}}\left(r_{\alpha \beta}\right) \theta_{\alpha \beta} \Delta t^{-1 / 2} \widehat{\mathbf{r}}_{\alpha \beta}$

where $\mathbf{v}_{\alpha \beta}=\mathbf{v}_{\alpha}-\mathbf{v}_{\beta}, \gamma$ is the friction coefficient, $\sigma$ is the noise amplitude, $\omega^{\mathrm{D}}\left(r_{\alpha \beta}\right)$ and $\omega^{\mathrm{R}}\left(r_{\alpha \beta}\right)$ are weight functions vanishing for $r>r_{\mathrm{c}}$ that describe the range of the dissipative and random forces, and $\theta_{\alpha \beta}$ is a randomly fluctuating variable with Gaussian statistics

$\left\langle\theta_{\alpha \beta}\right\rangle=0,\left\langle\theta_{\alpha \beta}(t) \theta_{k l}\left(t^{\prime}\right)\right\rangle=\left(\delta_{\alpha k} \delta_{\beta l}+\delta_{\alpha l} \delta_{\beta k}\right) \delta\left(t-t^{\prime}\right)$ 
The system should satisfy the fluctuation-dissipation theorem and evolve to an equilibrium state corresponding to the canonical ensemble. Therefore, only one of $\omega^{\mathrm{D}}\left(r_{\alpha \beta}\right)$ and $\omega^{\mathrm{R}}\left(r_{\alpha \beta}\right)$ can be chosen arbitrarily and the other one is then fixed by the relation

$\omega^{\mathrm{D}}\left(r_{\alpha \beta}\right)=\left[\omega^{R}\left(r_{\alpha \beta}\right)\right]^{2}=\omega\left(r_{\alpha \beta}\right)$

And the values of parameters $\gamma$ and $\sigma$ are coupled by

$\sigma^{2}=2 \gamma k_{B} T \Delta t$

where $k_{B}$ and $T$ are the Boltzmann constant and temperature, respectively.

The PBLG- $b$-PEG block copolymers were modeled by a coarse-grained linear polymer chain consisting of a rigid block and a flexible block. The PS sphere is represented by the crosslinked homopolymer sphere created by random covalent bonds between neighboring homopolymer chains. The neighboring beads in each block copolymer are connected by spring bonds given by $\mathbf{F}_{i j}^{\mathrm{S}}=-C^{\mathrm{S}}\left(r_{i j}-r_{e q}\right) \hat{\mathbf{r}}_{i j}$, where $C^{S}$ and $r_{e q}$ denote the spring constant and equilibrium bond length, respectively. An angle force, expressed as $\mathbf{F}_{i j}^{\theta}=-\nabla\left[k_{\theta}(\cos \theta-\cos \pi)^{2}\right]$, is applied to the rigid blocks to constrain the bond angle near $\pi$. In this work, the spring constant is set as $C^{S}=100$ and angle constant is $k_{\theta}=50$. The equilibrium bond distances $r_{e q}$ for $\mathbf{F}_{i j}^{S}$ is set to 0.7 .

\subsection{Simulation parameters}

All the DPD simulations are performed in a $80 \times 80 \times 80$ simulation box, where NVT ensemble and periodic boundary conditions are adopted. The particle density $\rho$ is set to 3 . The friction coefficient $\gamma$ and the noise amplitude $\sigma$ are respectively set to 4.5 and 3.0, and thus $k_{B} T=1.0$. In the simulation, more than $2 \times 10^{7} \mathrm{DPD}$ steps are performed so that the computing time is long enough for the system to achieve an equilibrium state. 
The bead numbers in the rod and coil block were chosen appropriately so that their relative length matched the block copolymer in experiments. For the $\mathrm{PBLG}_{29.5 \mathrm{k}}-b-\mathrm{PEG}_{5 \mathrm{k}}$ block copolymer used in our experimental system, the degrees of polymerization of the PBLG and each PEG blocks were $N_{\mathrm{PBLG}}=135$ and $N_{\mathrm{PEG}}=113$, respectively. Therefore, the relative number of DPD beads for rod and coil blocks should be set as 135:113 $\approx 1.2: 1$. As a result, the model of $\mathrm{R}_{6} \mathrm{C}_{5}$ rod-coil block copolymers composed of 6 rod $\mathrm{R}$ beads and 5 coil $\mathrm{C}$ beads was adopted in the study. Each beads in the RC block copolymer consist of 23 monomers.

The interaction parameters between different type of beads are listed in Table S2. The R, $\mathrm{C}, \mathrm{P}$ and $\mathrm{S}$ in the table are correspbonding to the rod beads, coil beads, beads in microspheres, and solvent beads, respectively. The parameters were set according to the experiment conditions. The interaction parameter between the R blocks and the P sphere $\left(a_{\mathrm{RP}}\right)$ is set as 25 to guarantee the adsorption of RC BCPs on the spheres. Since the rod blocks are solvophobic, the interaction parameter between R blocks and solvents $\left(a_{\mathrm{RS}}\right)$ is set to be 70 . Under these settings, the RC block copolymers are capable of assembling on the $\mathrm{P}$ sphere into surface micelles.

Table S2 Interaction Parameters $a_{\mathrm{ij}}$ (in DPD units) Used in the Simulations

\begin{tabular}{ccccc}
\hline & R (PBLG) & C (PEG) & P (PS sphere) & S (Solvent) \\
\hline R (PBLG) & 25 & 100 & 25 & 70 \\
C (PEG) & 100 & 25 & 30 & 25 \\
P (PS sphere) & 25 & 30 & 15 & 200 \\
S (Solvent) & 70 & 25 & 200 & 25 \\
\hline
\end{tabular}




\section{Simulations for Effect of Rod Block Length on Surface Nanostructure}

The effect of rod block length of RC block copolymers on the surface nanostructure was studied by varing the rod block length from 5 to 7 (Figure S8). Figure S8a, c and e show the the surface patterns self-assembled by $\mathrm{R}_{5} \mathrm{C}_{5}, \mathrm{R}_{6} \mathrm{C}_{5}$, and $\mathrm{R}_{7} \mathrm{C}_{5}$ on spherical substrates, respectively. Figure S8b, d and f are 2D Winkel Tripel projections of Figure S8a, c and e, respectively. As can be seen, dot-like surface micelles were obtained in all the systems.
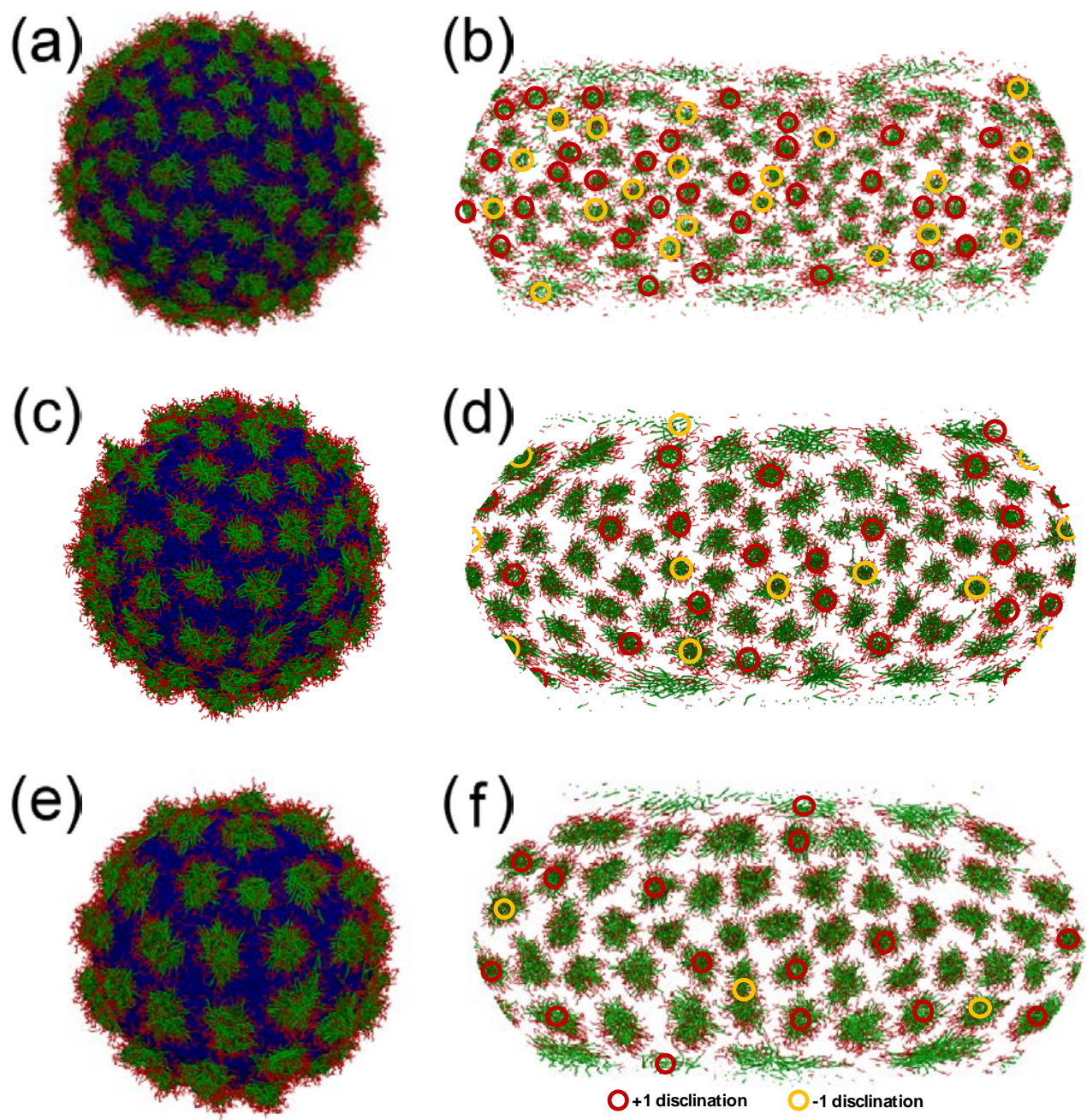

Figure S8. Simulation prediction for the surface-patterned particles and corresponding 2D projections formed by various RC model block copolymers on the model micorspheres: $(a, b) \mathrm{R}_{5} \mathrm{C}_{5}$; $(c, d) \mathrm{R}_{6} \mathrm{C}_{5}$; $(\mathrm{e}, \mathrm{f}) \mathrm{R}_{7} \mathrm{C}_{5}$. Solvents are not shown in these figures. 
To analysize the array feature of dot-like surface micelles with various length of rod block, we counted number of surface micelles on the microspheres. It was found that the 6-fold coordination is the majority array type for the surface micelles in all the systems, and the number of both 5-fold coordinated and 7-fold coordinated micelles increases as the length of rod blocks decreses. Furthermore, the total excess disclination number is 12, which is consistent with the theoretical value. The details regarding the array feature of the surface micelles formed by various RC BCPs are summarized in Table S3.

Table S3 Array feature of surface micelles formed by RC with various R block length

\begin{tabular}{ccclcc}
\hline $\begin{array}{l}\text { R block } \\
\text { length }\end{array}$ & $\begin{array}{l}\text { Number of } \\
\text { the surface } \\
\text { micelles on } \\
\text { each sphere }\end{array}$ & $\begin{array}{l}\text { 6-fold } \\
\text { coordinated } \\
\text { surface micelles }\end{array}$ & $\begin{array}{l}\text { 5-fold } \\
\text { coordinated } \\
\text { surface micelles } \\
(+1 \text { disclination })\end{array}$ & $\begin{array}{l}\text { 7-fold } \\
\text { coordinated } \\
\text { surface micelles } \\
(-1 \text { disclination })\end{array}$ & $\begin{array}{l}\text { Total excess } \\
\text { disclination } \\
\text { number }\end{array}$ \\
\hline 5 & 111 & 59 & 32 & 20 & 12 \\
6 & 76 & 46 & 21 & 9 & 12 \\
7 & 56 & 38 & 15 & 3 & 12 \\
\hline
\end{tabular}




\section{References}

(1) Zhuang, Z.; Jiang, T.; Lin, J.; Gao, L.; Yang, C.; Wang, L.; Cai, C. Hierarchical Nanowires Synthesized by Supramolecular Stepwise Polymerization. Angew. Chem. Inter. Ed. 2016, 55, 12522-12527.

(2) Cai, C.; Lin, J.; Zhu, X.; Gong, S.; Wang, X.-S.; Wang, L. Superhelices with Designed Helical Structures and Temperature-Stimulated Chirality Transitions. Macromolecules 2016, 49, 15-22.

(3) Yang, C.; Gao, L.; Lin, J.; Wang, L.; Cai, C.; Wei, Y.; Li, Z. Toroid Formation through a Supramolecular “Cyclization Reaction” of Rodlike Micelles. Angew. Chem. Int. Ed. 2017, $56,5546-5550$.

(4) Yuan, W.; Zhang, J.; Wei, J.; Zhang, C.; Ren, J. Synthesis and Self-Assembly of pH-Responsive Amphiphilic Dendritic Star-Block Terpolymer by the Combination of ROP, ATRP and Click Chemistry. Eur. Polym. J. 2011, 47, 949-958.

(5) Matyjaszewski, K.; Xia, J. Atom Transfer Radical Polymerization. Chem. Rev. 2001, 101, 2921-2990.

(6) Zhang, S.; Chen, J.; Taha, M. Synthesis of Monodisperse Styrene/Methyl Methacrylate/Acrylic Acid Latex Using Surfactant-Free Emulsion Copolymerization in Air. J. Appl. Polym. Sci. 2009, 114, 1598-1605.

(7) Zhang, S.; Zhu, Y.; Hua, Y.; Jegat, C.; Chen, J.; Taha, M. Stability of Surfactant-Free High Internal Phase Emulsions and Its Tailoring Morphology of Porous Polymers Based on The Emulsions. Polymer 2011, 52, 4881-4890. 
(8) Cai, C.; Li, Y.; Lin, J.; Wang, L.; Lin, S.; Wang, X.-S.; Jiang, T. Simulation-Assisted Self-Assembly of Multicomponent Polymers into Hierarchical Assemblies with Varied Morphologies. Angew. Chem. Int. Ed. 2013, 52, 7732-7736.

(9) Kim, K. T.; Park, C.; Vandermeulen, G. W. M.; Rider, D. A.; Kim, C.; Winnik, M. A.; Manners, I. Gelation of Helical Polypeptide-Random Coil Diblock Copolymers by a Nanoribbon Mechanism. Angew. Chem. Inter. Ed. 2005, 44, 7964-7968.

(10)Renka, R. J. Algorithm 772: STRIPACK: Delaunay Triangulation and Voronoi Diagram on the Surface of a Sphere. ACM Trans. Math. Softw. 1997, 23, 416-434.

(11)Zhang, L.; Wang, L.; Lin, J. Defect Structures and Ordering Behaviours of Diblock Copolymers Self-Assembling on Spherical Substrates. Soft Matter 2014, 10, 6713-6721.

(12)Zhang, Q.; Lin, J.; Wang, L.; Xu, Z. Theoretical Modeling and Simulations of Self-Assembly of Copolymers in Solution. Prog. Polym. Sci. 2017, 75, 1-30.

(13)Jiang, T.; Wang, L.; Lin, J. Mechanical Properties of Designed Multicompartment Gels Formed by ABC Graft Copolymers. Langmuir 2013, 29, 12298-12306.

(14)Xu, Z.; Lin, J.; Zhang, Q.; Wang, L.; Tian, X. Theoretical Simulations of Nanostructures Self-Assembled from Copolymer Systems. Polym. Chem. 2016, 7, 3783-3811. 